\title{
A decade of AGILE: Closing remarks
}

\author{
Marco Tavani ${ }^{1,2,3}$
}

Received: 21 October 2019 / Accepted: 25 October 2019 / Published online: 19 November 2019

(c) Accademia Nazionale dei Lincei 2019

This volume is an important achievement of the AGILE space project, being an excellent representation of the scientific and technical contributions of the Team and of the international community to gamma-ray astrophysics during the last years. A number of crucial scientific results have been obtained since the AGILE launch in 2007, and the Mission has always been at the forefront of exciting observations and difficult investigations.

The gamma-ray universe is rich in challenging phenomena and surprises. The AGILE Symposium held in December 2017 at the Accademia Nazionale dei Lincei provided an occasion to discuss the newest observations and present the last frontier of knowledge.

The Silicon detector technique at the heart of the AGILE gamma-ray measurements in space is an achievement of experimental particle physics, and Italian laboratories have been leading the path towards innovation for decades. The first talks and contributions in this volume summarize the path towards the current state-of-the-art, and demonstrate the strong link between particle physics and astrophysics. Today, the AGILE and Fermi groups and associated community lead the development for new challenging space missions (e-ASTROGAM, AMEGO) that may become real in the near future.

The exciting field of gravitational wave sources has been one of the first topics discussed at the Symposium. The

This paper is an Editorial of the Topical Collection originated by the Conference on Gamma Ray Astrophysics with the AGILE Satellite held at Accademia Nazionale dei Lincei and Agenzia Spaziale Italiana, Rome on December 11-13, 2017.

Marco Tavani

marco.tavani@inaf.it

1 INAF, Istituto di Astrofisica e Planetologia Spaziali, via Fosso del Cavaliere 100, I-00133 Rome, Italy

2 Dipartimento di Fisica, Università di Roma Tor Vergata, via della Ricerca Scientifica 1, I-00133 Rome, Italy

3 Accademia Nazionale dei Lincei, via della Lungara 10, I-00165 Rome, Italy
LIGO discovery of gravitational signals from black holeblack hole coalescences started in 2015 an entirely new chapter of Astronomy. Today, the focus is not only on the GW events by themselves, but also on the search for electromagnetic counterparts. The neutron star-neutron star GW event of August 17, 2017 was followed by a detection of a feeble hard X-ray event lasting a couple of seconds, as observed by the Fermi GBM detector and by Integral. This event was later associated with a optical and radio (and later $\mathrm{X}$-ray) fading source of a new type, with the characteristics of a so-called "kilonova". AGILE was occulted by the Earth shadow at the time of this GW event; however, it contributed to the first gamma-ray imaging of the field containing the event position after about $1000 \mathrm{~s}$, excluding the existence of a powerful "magnetar". This event, so far unique at the time of this writing, constitutes a milestone for future research. More associable transients of this type are needed to secure the link with GW events. The contributions at the Symposium emphasized the challenging searches for GW source counterparts, and reported on the observational and theoretical investigations dealing with the exotic realm of remote coalescences of compact objects.

A classic topic of gamma-ray astrophysics deals with pulsars, i.e., rotationally powered neutron stars with magnetic fields. We know today many hundreds of gamma-ray pulsars, and the Symposium presentations focused on new theoretical models for gamma-ray emission that today favor magnetic field reconnection at the boundary of the pulsar magnetosphere as an important contribution to the pulsar electrodynamics.

The topic of relativistic pulsar winds and the physical processes involved in the interaction with their nebular environments is of the greatest relevance. Indeed, the AGILE discovery of fast gamma-ray flares from the Crab Nebula (a prototypical pulsar at the center of a supernova remnant left by a star exploded almost 1000 years ago) demonstrated that a novel particle acceleration mechanism occurs in the system. Since the AGILE discovery, this issue is at the center of many theoretical investigations on ways to efficiently accelerate particles at the highest energies up to 
$10^{15} \mathrm{eV}$. Impulsive magnetic reconnection of sheared patterns of field lines is a likely possibility, and several studies are now focused to strengthen the link between relativistic plasma physics and the gamma-ray astrophysics of the Crab Nebula flares. Exciting developments will follow from these investigations, for sure.

Galactic gamma-ray sources (different from pulsars) constitute a well defined set of objects in our Galaxy with still mysterious properties. Binary systems containing accreting compact objects (such as Cygnus $X-1$ and Cygnus $X-3$ ) or non-accreting systems (such as the binary pulsar PSR 125963 and possibly the famous gamma-ray source $2 C G 135+1 /$ $L S I+61303$ ) have been thoroughly observed by AGILE over the years, and interesting phenomena have been discovered. Among the best established patterns is the determination in Cygnus X-3 of a recurrent sequence of X-ray transitions leading to gamma-ray flares above $100 \mathrm{MeV}$ in conjunction with relativistic jet emission. This surprising phenomenon, discovered by AGILE at the beginning of its observations in 2008, has been detected several times during the last decade, and represents the best way to study the preparation and the final occurrence of a relativistic jet emission from a compact object.

AGILE contributed greatly to the study of Active Galactic Nuclei that emit enhanced gamma-ray fluxes because of the special geometry of their relativistic jets pointing towards the Earth (called "blazars"). Several contributions to the Symposium focused on the fundamental discoveries made in recent years that lead to a deeper view (compared to the pre-AGILE era) of the physical processes operating in these jets. The interplay between gamma-rays and the rest of the electromagnetic spectrum has been at the center of many observational and theoretical investigations. It is clear today that there is a number of gamma-ray flares from blazars that are strongly linked in time with optical and X-ray emissions. On the other hand, there is a very remarkable class of flares for which the gamma-ray emission appears to be surprisingly decoupled from the optical and X-ray emissions. This fact is highly problematic in a scenario based on gamma-rays radiated by inverse Compton emission in the inner regions near the central black hole for which high-density photon regions can be available. Modelling the "Compton dominance" of a class of blazars' flares is a challenging task for theoretical studies, and several alternatives are being proposed as reported at the Symposium. Other topics presented in the Symposium were the properties of blazars of the "BL Lac" class and the possible connection of high-energy neutrinos with the gamma-ray flaring of blazars. The latter point, if consolidated by more detections, would link the production of cosmic neutrinos with the acceleration of ultra-relativistic cosmic rays in blazars's jets, a possibility that awaits new observations to be confirmed. AGILE observations are interesting in this framework, and promising.
The origin of Galactic cosmic rays (CRs) has been for decades one of the most challenging problems of physics, and recent observations led to great progress. AGILE first unambiguous determination of a neutral pion decay signature in the spectrum of the Supernova Remnant (SNR) W44 demonstrated that this class of systems accelerate hadrons up to hundreds of GeV. AGILE and Fermi observations of SNRs indicate that hadrons can indeed be accelerated at shock fronts. The challenge is now to show how CRs can reach PeV energies and beyond. The search of "Pevatrons" is then a leading theme of high-energy astrophysics today, and the focus is on young SNRs such as Tycho or Cas A. In addition to SNRs, also shocks in the interstellar medium produced in young star clusters with strong interstellar winds are being considered today, and several contributions to the Symposium focused on this latter possibility. If demonstrated, a variety of conditions can be relevant to the CR acceleration, involving energetic SNRs and interstellar shocks. Investigating the physics underlying these processes is fascinating.

Gamma Ray Bursts (GRBs) have attracted considerable attention since their discoveries in the 1970s. These transient events are detected in the hard X-ray spectral region, and they may have emissions up to $\mathrm{GeV}$ energies and beyond in particular cases. AGILE and Fermi detected a large number of GRBs above $100 \mathrm{MeV}$, and the study of these events has very important theoretical implications. The interplay between synchrotron and inverse Compton emissions is dramatically displayed in the rapid GRB spectral evolution, both during the early "prompt" phase, and later during the delayed "afterglow" phase. The contributions to the Symposium summarized the current observations and the theoretical prospects for a deeper understanding of acceleration and radiative properties of these very energetic events.

Last but not least among the scientific results of AGILE is its contribution to the study of Terrestrial Gamma-Ray Flashes (TGFs). These millisecond flashes of gamma rays are produced by lightning associated with intense storms in the equatorial region. AGILE provides unique data for the study of TGFs because of its energy range (extending up to $100 \mathrm{MeV}$ ) and on-board millisecond trigger capability. An interesting sequence of phenomena may occur in the atmosphere linking TGFs with the production of secondary particles and gamma rays that can be detected on the ground, as demonstrated by the contributions to this volume.

The last session of the Symposium was devoted to the AGILE Mission technical "pillars" and the consequent legacy for the future. Indeed, not only the instrument but also its peculiar data handling system, on-board software, Ground Segment operations and the very fast analysis at the AGILE Data Center are to be considered as a basis for his success over the years. Several contributions at the Symposium presented the AGILE way of operating in space and on 
the ground with a particular emphasis on the lessons learned. AGILE indeed is technically a Small Mission but its legacy will extend well beyond the boundaries of the groups that participated in its development and operations.

This volume is dedicated to the many scientists and engineers who made possible the AGILE Mission. We acknowledge the cooperation and hard work of ASI, INAF, INFN, CNR, CIFS, and Italian Universities. At the time of this writing (October 2019) AGILE is in its thirteenth year in space with nominal performance, and is still collecting gamma-ray data from the cosmos. Let us persevere in this endeavour without anticipating now the surprises and new results that the Mission will continue to provide.

Publisher's Note Springer Nature remains neutral with regard to jurisdictional claims in published maps and institutional affiliations. 